\title{
Effect of Mulligan Bent Leg Raise versus Neural Mobilization on Hamstring Tightness in College Students
}

\author{
Shrinivas Vinayakrao Shinde ${ }^{1}$, Smita Bhimrao Kanase ${ }^{2}$ \\ ${ }^{I}$ (Intern, Faculty of Physiotherapy, Kimsdu Karad, Dist: Satara, Maharashtra, India) \\ ${ }^{2}$ (Lecturer, Faculty of Physiotherapy, Kimsdu, Karad, Dist: Satara, Maharashtra, India)
}

\begin{abstract}
Objective: The purpose of this study was to evaluate the immediate effect of mulligan bent leg raise and neural mobilization on hamstring tightness in college going students. Methods: This experimental study was conducted in Krishna institute of medical sciences, faculty of Physiotherapy, karad. Fifty students (aged 1825 years) with hamstring tightness were randomly assigned to 2 groups. Active knee extension and straight leg raise was measured using universal goniometer. These variables were assessed before and after intervention. Group A $(n=25)$ received Mulligan bent leg raise and Group B $(n=25)$ received neural mobilization technique. Results: Statistically extremely significant improvement in active knee extension and straight leg raise was observed within the groups. But between groups analysis showed significant and very significant improvement in Group B than Group A. Conclusion: Mulligan bent leg raise and neural mobilization techniques both showed immediate improvement in decreasing hamstring tightness which was reflected by increase in active knee extension and straight leg raise. But between groups comparison showed neural mobilization to be more effective than mulligan Bent leg raise technique
\end{abstract}

Keywords: Hamstring, mulligan bent leg raise, neural mobilization, and tightness.

\section{Introduction}

Hamstring muscles located on posterior compartment of thigh comprises of three large muscles namely semitendinosus, semimembranosus and biceps femoris. The muscles causes hip extension and knee flexion indicating that it is one of the two joint muscle of the body. When it comes to the injury rate within hamstring muscle, it is the long head of biceps femoris that is frequently injured and accounts for approximately $80 \%$ of all hamstring injuries.[1-3]

The most important component of physical fitness is flexibility. Normal flexibility enables the person to move smoothly and safely. For normal locomotion flexibility of muscle is important. It will help for normal body function. The flexibility of hamstring muscle is always emphasized more.[4-6]

The commonest muscle to always go for tightness is the Hamstring muscle. The tightness of this muscle apparently increases from childhood up to the age of 40-49 years. The incidence of tightness of hamstring muscle is higher in males than females. [7-8]

When hamstring muscle undergoes tightness it causes a profound effect on body's normal postural alignment. Till date studies have shown that tightness of hamstring always leds to low back pain with lumbar intervertebral disc pathologies. Biomechanically tightness of hamstring muscle is thought to increase patellofemoral joint compressive forces because of increase in passive tension at the time of swing phase of ambulation. [9-11] Under rapid and stressful situations hamstring muscles fails to pass through full physiological amplitude when specifically this muscle goes for tightness. [12]

Normal hamstring flexibility is affected by numerous factors which includes age, gender, race, tissue temperature, strength training, stiffness, awkward posture and reduced warm up period during exercise.[13] Various Physiotherapy treatment techniques like manual therapy and electrical agents are available to treat hamstring tightness. The techniques namely includes various types of stretching techniques, muscle energy technique, position release technique, myofacial release techniques.

Mulligan Bent Leg Raise is a newer technique which is recently been developed to manage hamstring tigthness. It is a painless stretching technique which is recently utilized in management of hamstring tightness with limitation of straight leg raise. [14] When the normal flexibility is affected and the hamstring muscle goes for tightness ther is always an alteration in neural tissue mobility which is also called as altered neurodynamics which causes enhanced neural mechanosensitivity. It is also been stated that decrease hamstring flexibility could be due to altered neurodynamics affecting the sciatic and tibial nerve.

The neural mobilization or nerve glide stretches is an neurodynamic intervention which are given as active stretches through which the nervous system is made taut and then slack this intervention decreases neural mechanosensitivity by providing movement which changes the neurodynamics and modifies sensation, and helps to explain the observed increase in flexibility. [15] 
Effect of Mulligan Bent Leg Raise versus Neural Mobilization on Hamstring Tightness in College ..

The dynamic balance between the relative movement of neural tissues and surrounding mechanical interfaces is maintained by neural mobilization. It reduces intrinsic pressures on the neural tissues and promotes optimum physiologic function [16] and it is assumed possible that if this techniques will be include in management of hamstring flexibility it can provide some beneficial effect.

Thus, the present study is conducted with an aim to find out best technique which will help in improving the hamstring flexibility and decrease the muscle tightness.

\section{Methods}

\subsection{Study population:}

College students of KIMSDU campus with hamstring tightness in lower limb having active knee extension less than 160 degrees were recruited in the study. It was opted to choose only college students as hamstring tightness is more prevalent from childhood to age 40-49 years. Theinclusion criteria for the study wasboth male and female participants willing to participate in the study, age group between 18-25 years, active knee extension less than 160 degrees. The exclusion criteria of study wasfractures of lower limb, individuals with any soft tissue injury of lower limb, individuals having chronic back pain, subjects having PIVD. The ethical clearance for the study was taken from institutional ethical committee of KIMSDU, Karad. All subjects signed an informed consent before the commencement of interventions.

\subsection{Study design:}

All subjects diagnosed with hamstring tightness at KIMS hospital, KARAD who reported to Physiotherapy department of KIMS hospital volunteered to participate in the study were selected as subjects. Each of the subject was screened as per inclusion and exclusion criteria and they were be briefed about the study and intervention. Informed consent was taken from the subjects.

Initial thorough assessment of each subject was taken as per data collection sheet. Active knee extension range, SLR range with goniometer was taken pre interventional and immediate post intervention.

A total no. of 50 subjects were divided into two groups with simple lottery method (25 subjects in each group). Group A included males and females treated with Mulligan Bent Leg raise technique and Group B included males and females treated with Neural Mobilization technique.

\subsection{Outcome measures:}

1. Active knee extension.

2. Straight leg raise.

\subsection{Intervention:}

Participants were randomly allocated to either group A treated with mulligan bent leg raise or group B treated with neural mobilization using simple lottery method.

Both the group participants were given 15 minutes of hot moist fermentation over the posterior aspect of leg prior to specific intervention.

GROUP A (MULLIGAN BLR): Subject was in supine lying position with knee flexed to 90 degrees and placed over the therapist shoulder. The popliteal fossa of knee rested on therapist shoulder. The subject was then asked to push therapist away with his/her flexed leg and then relax voluntarily.At this point therapist pushed subjects bent knee up as far as possible, in the direction of the subjects shoulder on the same side increasing hip flexion of the subject provided there was no pain. Subject was asked to hold each hamstring muscle isometric contraction for 6 seconds and 3 repetitions were performed which were pain free. Alongitudinal traction force along long axis of femur was also added with this technique.

GROUP B (NEURAL MOBILISATION):Subject was in supine lying position and was asked to place the leg on therapists shoulder. The leg was lifted with knee in extension to a point where subject felt the mild stretch in hamstring, when a mild stretch was felt therapist rotated hip medially and the foot was maintained in dorsiflexion. This position was maintained for $6 \mathrm{sec}$ and the leg was brought to resting position. 3 repetitions were performed.

\subsection{Statistical analysis:}

Data were analyzed using instat software. Descriptive statistics (mean \pm standard deviation) were calculated for all parameters. The $\mathrm{p}$ value $<0.0001$ was considered extremely statistically significant.

\section{Results}

Fifty participants (age range, 18-22 years) participated in this study. Twenty-five subjects were allocated to group A, whereas 25 participants were included in group B.demographic data of both the groups are summarized in ("table" 1).Group A included males and females treated with Mulligan Bent Leg raise technique and Group B included males and females treated with Neural Mobilization technique. 
Effect of Mulligan Bent Leg Raise versus Neural Mobilization on Hamstring Tightness in College ..

Descriptive statistics of primary outcome measures are shown in table 2 . The paired t test analysis was done to analyze the data within the groups. The unpaired t test was done to analyze the data between the 2 groups

$>$ Active knee extension on right side:(“table" 2)

The paired t test analysis revealed a significant improvement in active knee extension of right lower extremity within the group A and Group B. post treatment there was increase in active knee extension of right lower extremity $(\mathrm{p}<0.0001)$

$>$ Active knee extension on left side:("table" 2)

The paired t test was used to analyse the data for active knee extension of left lower extremity within the group A and Group B. the post treatment values revealed statistically significant increase in active knee extension range of left knee joint in both the groups. $(p<0.0001)$

$>$ Straight leg raise right side:(“table" 2)

The within group analysis done using paired t test showed statistically extremely significant improvement in post treatment values of straight leg raise range of motionon right side in both the groups $(\mathrm{p}<0.0001)$

$>$ Straight leg raise left side: ("table" 2)

The within group analysis done using paired t test showed statistically extremely significant improvement in post treatment values of straight leg raise range of motion on left side in both the groups $(\mathrm{p}<0.0001)$

\section{Between group statistical analysis:}

$>$ Active knee extension right side:("table" 3)

The between group analysis for active knee extension on right side revealed no significant difference pre intervention $(\mathrm{p}=0.1144)$. the post intervention values revealed statistically very significant improvement in active knee extension range of right side in group $B$ than in Group $A(p=0.0046)$. this was done using unpaired t test

$>$ Active knee extension left side:("table" 3)

The between group analysis done using unpaired t test for active knee extension on left side revealed no significant difference pre intervention( $\mathrm{p}=0.2373)$. the post intervention values revealed statistically significant improvement in active knee extension range of right side in group $B$ than in Group $A(p=0.0241)$

$>$ Straight leg raise right side: ("table" 3 )

The between group analysis done using unpaired t test for Straight leg raise on right side revealed no significant difference pre intervention $(\mathrm{p}=0.7940)$. the post intervention values revealed statistically significant improvement in Straight leg raise on right side in group B than in Group $A(p=0.0186)$

$>$ Straight leg raise left side: ("table" 3 )

The between group analysis done using unpaired t test for Straight leg raise on left side revealed no significant difference pre intervention $(\mathrm{p}=0.2330)$. the post intervention values revealed statistically no significant improvement in Straight leg raise on left side in group B than in Group A(p=0.1771)

\section{Discussion}

The aim of this study was to determine whether mulligan bent leg raise is better than neural mobilization in improving hamstring tightness in college students. The effect was ruled out by measuring active knee extension range and straight leg raise range on involved side. The second aim was to rule out the immediate effects of both the techniques on hamstring tightness.

It was hypothesized that there will be no significant differences in both the techniques on hamstring tightness. Improvement after the treatment with mulligan bent leg raise or neural mobilization were found on immediate basis for active knee extension and straight leg raise range of motion measured on bilateral lower limb. The active knee extension and straight leg raise of right and left side was statistically extremely significantly improved in group A participants treated with mulligan bent leg raise. This improvement is mainly attributed to change in stretch tolerance of hamstring muscle. Goeken and Hof has described the increased SLR following this technique is through increase in range of hip flexion and the length of hamstring muscle. [17]

It may also be assumed that bent leg raise triggers neurophysiological responses influencing the muscle stretch tolerance. [14-17] Thus, there was improvement in active knee extension and straight leg raise range in participants of group A treated with Mulligan Bent Leg raise which correlates with previous studies done.

The participants of group B treated with neural mobilization also showed statistically extremely significant improvement in active knee extension and straight leg raise range immediate post interventionally. The improvement is this Group is mainly due to improvement in neurodynamics, axoplasmic flow and facilitation of maintaining the dynamic balance between neural tissue and surrounding mechanical interface and in turn dampening the mechanosensitivity.[16-18]

When it comes to between group comparision of values post interventinally it was found that their was very significant and significant improvement in active knee extension of right side and active knee extension of left side, straight leg raise of right side respectively in group B treated with neural mobilization than in Group A 
Effect of Mulligan Bent Leg Raise versus Neural Mobilization on Hamstring Tightness in College ..

treated with Mulligans bent leg raise. Whereas the improvement in straight leg raise of left side was equal in both the groups with no statistically significant difference.

Thus, the above study findings showed neural mobilization to be effective statistically then mulligan bent leg raise in improving active knee extension and straight leg raise.

\section{Limitations:}

The limitations of the study Small sample size, Short study duration, and Limited literature review on use of neural mobilization on hamstring tightness. In this study subject were not homogenous.

\section{Conclusion}

Neural mobilization was found to be more effective than mulligan bent leg raise in treating the hamstring tightness in college students.

\section{Acknowledgements}

Authors thank all the people who have directly or indirectly contributed and helped to conduct the research.

\section{References}

[1] AkinpeluAo ,Bakareu, Adegokeboa. Influence of age on hamstring tightness in apparently healthy Nigerians: Journal of the Nigeria Society of Physiotherapy Vol 115: 35 - 41, (2005).

[2] B Dadebo, J White, K P George. A survey of flexibility training protocols and hamstring strains in professional football clubs in England: British Journal of Sports Medicine 2004; Vol 38: 388-394.

[3] Michael R.Rehorn, Silvia S.Blemker: The effects of aponeurosis geometry on strain injury susceptibility explored with a 3D muscle model: Journal of Biomechanics 43 (2010) 2574-2581.

[4] Odunaiya NA, Hamzat TK, Ajayi OF. The effects of static stretch duration on the flexibility of hamstring muscles. Journal of biomedical research. 2005; 8: 79-82.

[5] Nagarwal AK, Zutshi K, Ram CS and Zafar RI. mprovement of hamstring flexibility: A comparison of two PNF stretching techniques. International journal of sports science and engineering, 2010; 04(01): 025-033.

[6] Brasileiro JS, Faria AF and Queiroz LL. Influence of local cooling and warming on the flexibility of the hamstring muscles. Rev bras. Fisioter Sao Carlos. 2007;11(1): 53-57.

[7] Turner D, Gossman RM., Nicholson CG and Lemons J. Comparison of cyclic and sustained passive stretching using a mechanical device to increase resting length of hamstring muscles. Physical Therapy 1988; 69: 314-20.

[8] Aderonke O, Bakere U, Adegoke BOA. Influence of age on hamstring tightness in apparently healthy Nigerians. J Nigsocphysiother 2005; 15: 35-41.

[9] Wendell L. Unresolved controversies in back management. J Orthopaedic Sports Physical Therapy 1988; 9: 1213-16.

[10] Christine E. Back pain re-visited. J Orthopaedic Sports Physical Therapy. 1987; 8: 230-34.

[11] Gaillet R. Low back pain syndrome. 3rd ed. Philadelphia Pa: 1980.

[12] Baer J. Iliotibial Band Syndrome in Cyclists: Evaluation and Treatment; A Case Report. J Sports Chiropractic Rehabilitation 1999; 13: 66-9.

[13] Fasen JM, Connor AM, Schwartz S, et al. A randomized controlled trial of hamstring stretching: comparison of four techniques. Journal of strength and conditioning research. 2009; 23(2): 660-667.

[14] Hall T, Hardt S, Schafer A, Wallin L. Mulligan bent leg raise technique--a preliminary randomized trial of immediate effects after a single intervention. Man Ther 2006;11(2):130-5

[15] Castellote-Caballero Y, Valenza MC, et al. Immediate effects of neurodynamic versus muscle stretching on hamstring flexibility in subjects with short hamstring syndrome. A pilot study. Physical therapy in sport. 2013;14(3), 156-162

[16] Ellis RF, Hing WA. Neural mobilization: A systematic review of randomized controlled trials with an analysis of therapeutic efficacy. J Man ManipTher. 2008; 16(1): 8-22.

[17] Goeken LN, Hof HL. Instrumrntal straight leg raising: results in patients. Archives of Physical Medicine and rehabilitation 1994; 75(4):470-7.

[18] Malik N., Kataria C., SachdevN.;comparative effectiveness of straight leg raise and slump stretching in subjects with low back pain with advers neural tension:international journal of health and rehabilitation sciences.(2012);1(1):2-10.

Tables

\begin{tabular}{|l|l|l|l|}
\hline & GROUP A & GROUP B & Total \\
\hline MALES & 11 & 13 & 24 \\
\hline FEMALES & 14 & 12 & 26 \\
\hline TOTAL & 25 & 25 & 50 \\
\hline Mean Age (years) & 21.04 years & 20.4 years & \\
\hline
\end{tabular}

Table 1: Demographic Data

\begin{tabular}{|c|c|c|c|c|}
\hline Outcome measure & Time point & Mean \pm SD & Within group differences & P value \\
\hline \multirow[t]{2}{*}{ AKE right group $\mathrm{A}$} & Pre treatment & $128.68 \pm 12.789$ & \multirow[t]{2}{*}{17.200} & \multirow[t]{2}{*}{$<0.0001$} \\
\hline & Post treatment & $145.88 \pm 13.007$ & & \\
\hline \multirow[t]{2}{*}{ AKE right group $\mathrm{A}$} & Pre treatment & $134 \pm 10.496$ & \multirow[t]{2}{*}{21.640} & \multirow[t]{2}{*}{$<0.0001$} \\
\hline & Post treatment & $155.64 \pm 10.004$ & & \\
\hline \multirow[t]{2}{*}{ AKE left Group A } & Pre treatment & $130.56 \pm 11.493$ & \multirow[t]{2}{*}{18.640} & \multirow[t]{2}{*}{$<0.0001$} \\
\hline & Post treatment & $149.2 \pm 12.25$ & & \\
\hline \multirow[t]{2}{*}{ AKE left Group B } & Pre treatment & $134.44 \pm 11.931$ & \multirow[t]{2}{*}{22.320} & \multirow[t]{2}{*}{$<0.0001$} \\
\hline & Post treatment & $156.76 \pm 10.631$ & & \\
\hline
\end{tabular}


Effect of Mulligan Bent Leg Raise versus Neural Mobilization on Hamstring Tightness in College ..

\begin{tabular}{|l|l|l|l|l|}
\hline \multirow{2}{*}{ SLR right group A } & Pre treatment & $41.9 \pm 7.188$ & 15.900 & $<0.0001$ \\
\cline { 2 - 3 } & Post treatment & $57.8 \pm 8.198$ & & \\
\hline \multirow{2}{*}{ SLR right group B } & Pre treatment & $40.96 \pm 9.838$ & 22.760 & $<0.0001$ \\
\cline { 2 - 3 } & Post treatment & $63.72 \pm 8.970$ & & \\
\hline \multirow{2}{*}{ SLR left group A } & Pre treatment & $43.8 \pm 6.801$ & 17.640 & $<0.0001$ \\
\cline { 2 - 3 } & Post treatment & $61.44 \pm 7.773$ & 23.800 & $<0.0001$ \\
\hline \multirow{2}{*}{ SLR left group B } & Pre treatment & $41 \pm 9.385$ & & \\
\cline { 2 - 3 } & Post treatment & $64.8 \pm 9.487$ & & \\
\hline
\end{tabular}

Table 2: Within Group Statistical Analysis

\begin{tabular}{|l|l|l|l|l|l|}
\hline \multirow{2}{*}{ Outcome measure } & Time & Mean \pm SD & Difference & P value \\
\cline { 3 - 4 } & & Group A & Group B & & \\
\hline \multirow{2}{*}{ AKE RIGHT } & Pre treatment & $128.68 \pm 12.780$ & $134 \pm 10.496$ & \multirow{2}{*}{9.760} & $0.1144 \mathrm{NS}$ \\
\cline { 2 - 4 } & Post treatment & $145.88 \pm 13.007$ & $155.64 \pm 10.004$ & & $0.0046 \mathrm{VS}$ \\
\hline \multirow{2}{*}{ AKE LEFT } & Pre treatment & $130.56 \pm 11.493$ & $134.44 \pm 11.931$ & \multirow{2}{*}{7.560} & $0.2473 \mathrm{NS}$ \\
\cline { 2 - 4 } & Post treatment & $149.2 \pm 12.258$ & $156.76 \pm 10.631$ & & $0.0241 \mathrm{~S}$ \\
\hline \multirow{2}{*}{ SLR RIGHT } & Pre treatment & $41.6 \pm 7.188$ & $40.96 \pm 9.838$ & 5.920 & $0.7940 \mathrm{NS}$ \\
\cline { 2 - 4 } & Post treatment & $57.8 \pm 8.190$ & $63.72 \pm 8.970$ & & $0.0186 \mathrm{~S}$ \\
\hline \multirow{2}{*}{ SLR LEFT } & Pre treatment & $43.8 \pm 6.801$ & $41 \pm 9.385$ & \multirow{2}{*}{3.360} & $0.2330 \mathrm{NS}$ \\
\cline { 2 - 4 } & Post treatment & $61.44 \pm 7.773$ & $64.8 \pm 9.487$ & & $0.1771 \mathrm{NS}$ \\
\hline
\end{tabular}

Table 3: Between group analysis 